\title{
Application of linear analysis in traction power system stability studies
}

\author{
S. Danielsen, T. Toftevaag \& O. B. Fosso \\ Department of Electric Power Engineering, \\ Norwegian University of Science and Technology, Trondheim, Norway
}

\begin{abstract}
Dynamical phenomena such as oscillations and instability in railway power systems have caused concern in the experts' community the recent years. On several occasions, modern advanced electrical rail vehicles have been the source of low frequency power oscillations leading to an unstable power system due to lack of damping, and as a consequence operational problems. A method to study these phenomena is needed. Well known linear techniques based on small-signal analysis provide valuable information about the inherent characteristics of even non-linear single-phase power systems. This paper describes how a traction power system and its dynamical railway-related components are modelled in a commercially available power system analysis software and studied by linear analysis such as eigenvalues, participation factors and parameter sensitivities. This is used to gain knowledge about the interaction between the rail vehicles and the electrical infrastructure. Linear analysis is found to be a powerful tool in this respect provided that adequate models of the relevant components can be established in RMS mode. The results clearly indicate poor interaction.

Keywords: AC railway power supply, traction power system, stability, advanced electrical rail vehicle, rotary converter, low frequency oscillations, eigenvalue analysis.
\end{abstract}

\section{Introduction}

The recent development of electrical rail vehicles with utilization of power electronics and complex control systems has introduced new phenomena of dynamical interaction between electrical rail vehicles and railway power supply. One such phenomenon is low frequency power oscillations leading to system 


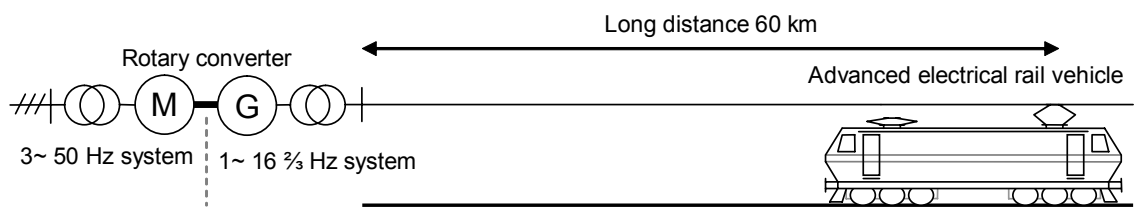

Figure 1: Principal sketch of the traction power system to be studied.

instability, typically in the frequency range of 0.1-0.3 times the fundamental frequency. The vehicles may oscillate together internally (Menth and Meyer [1]) or against the electrical infrastructure (Danielsen and Toftevaag [2]). There is a need for an integrated method for studying these oscillations.

Low frequency power oscillations are not new to power systems (Kundur [3]). One method to study these inherent qualities of the power system is by utilization of linear analysis. Several specialized power system analysis computer programs include such tools, i.e. Simpow (Fankhauser et al. [4]).

This paper introduces linear analysis to a traction power system study where the system comprises both electrical infrastructure and rolling stock as shown in Figure 1. An alternative method is time domain simulations as used in Eitzmann et al. [5].

\section{Linear analysis theory}

A dynamical system may be described by a number of characteristic differential equations, normally based on the physics of the system to be studied. Based on these equations and information about the initial conditions, the state of the system can be determined and the response of a disturbance can be calculated.

If the system is non-linear, commonly the system is linearized around an operation point ( $\Delta$-values). In that way the mathematical tools that are used for linear systems can be utilized for non-linear systems as well, such as traction power systems. This is formally only valid in vicinity of the linearization point.

A common way to describe a linear or linearized system is by a state space model as in eqn. (1) where $\mathbf{X}$ is the state vector containing the state variables, i.e. the variables of which the time derivative is to be considered, and $\mathbf{u}$ is the vector containing disturbance variables. $\dot{\mathbf{x}}$ is a vector containing the time derivative of the state variables and $\mathbf{y}$ is called the output vector.

$$
\begin{aligned}
\Delta \dot{\mathbf{x}} & =\mathbf{A} \Delta \mathbf{x}+\mathbf{B} \Delta \mathbf{u} \\
\Delta \mathbf{y} & =\mathbf{C} \Delta \mathbf{x}+\mathbf{D} \Delta \mathbf{u}
\end{aligned}
$$

Matrix $\mathbf{A}$ is the state matrix and contains important information about the inherent qualities of the linear/linearized system. The roots of the characteristic eqn. (2),$\lambda$, are called the eigenvalues of the system.

$$
\operatorname{det}(\mathbf{A}-\lambda \mathbf{I})=0
$$

The number of eigenvalues for a system is equal to the dimension of $\mathbf{A}$ and the number of first order differential equations describing the system. An 
eigenvalue can be a complex number $\lambda=\sigma+j \omega$ describing a mode of the system where the imaginary part describes the oscillation frequency and real part describes the damping or time decay of the oscillation. A negative real part identifies a stable mode. Complex eigenvalues appear as conjugate pairs, but only the eigenvalue having positive imaginary part is shown in the figures in this paper.

The concept of participation factors utilizes information from the state matrix to find a measure for the relative participation of a specific state variable in a specific mode. The factor itself is a phasor where its length reflects the relative degree of participation in the mode compared to other state variables and the angle describes how it contributes in the specific mode, in phase or in anti-phase.

Linear analysis of large power systems requires a lot of computations, but it is a simple task for a computer with suitable software (Slootweg et al. [6]).

\section{Concept of power system modelling}

In an $\mathrm{AC}$ power system, both voltage and current vary as a sine with the fundamental frequency $f_{1}$ (such as $16^{2} / 3 \mathrm{~Hz}$ or $50 \mathrm{~Hz}$ ) plus additional harmonics. The voltage drop $\Delta u(t)$ given by a current $i(t)$ over an inductive impedance is described by Ohm's law as in eqn. (3).

$$
\Delta u(t)=R \cdot i(t)+L \frac{d i(t)}{d t}
$$

A common simplification for power system analysis is to represent voltages and currents by phasors, i.e. $\Delta \vec{U}=\Delta U_{\mathrm{Re}}+j \Delta U_{\mathrm{Im}}$. The calculated voltage drop over the impedance will then depend on the resistance $R$ and the fundamental angular frequency $\omega_{1}=2 \pi f_{1}$ times the inductance $L$. This common simplification implies that the current is no longer a state variable where its time derivative is considered. In traditional power systems analysis, the impact of this simplification may be neglected in the view of stability (Kundur [3]). For analysis of power systems with power electronic converters such as an advanced electrical rail vehicle, this simplification may not be valid any more (Danielsen et al. [7]). In the present studies the current is therefore kept as a state variable giving the expression for the voltage drop of any series inductive impedance in the network as in eqn. (4).

$$
\left[\begin{array}{l}
\Delta U_{\mathrm{Re}} \\
\Delta U_{\mathrm{Im}}
\end{array}\right]=\left[\begin{array}{cc}
R+L \frac{d}{d t} & -\omega_{1} L \\
\omega_{1} L & R+L \frac{d}{d t}
\end{array}\right]\left[\begin{array}{l}
I_{\mathrm{Re}} \\
I_{\mathrm{Im}}
\end{array}\right]
$$

Keeping the current as a state variable is often used in studies of power electronic components in instantaneous value mode analysis of three-phase systems modelled in the rotating $d q$ reference frame (Harnefors [8]), but is here applied to an entire single-phase power system. The phasors are RMS values of the respective voltages and currents. i.e. root mean squared values over one fundamental frequency period, hence harmonic effects are neglected. 


\section{Rotary converter}

\subsection{Introduction}

The rotary converter is the dominant solution of electric power conversion from the three-phase $50 \mathrm{~Hz}$ utility grid to the single-phase $16^{2 / 3} \mathrm{~Hz}$ decentralized fed traction power system in Norway and Sweden (Banverket/Jernbaneverket [9]). Such a converter consists of a three-phase synchronous motor $(M)$ mounted on the same shaft as a single-phase synchronous generator $(G)$, see Figure 1. Both motor and generator are equipped with automatic voltage regulators and exciters.

\subsection{Electromechanical resonance}

A pronounced characteristic of these rotary converters is the poorly damped electromechanical resonance around $1.6 \mathrm{~Hz}$ due to the lack of explicit motor damper windings (Toftevaag and Pálsson [10]). These low frequency oscillations are shown to be related to the basic swing equation (Biesenack [11]). This equation is linearized in eqn. (5) and describes the electromechanical behaviour (which is characteristic for all synchronous machines (Kundur [3])):

$$
2 H \frac{d^{2} \Delta \delta}{d t^{2}}+D \frac{d \Delta \delta}{d t}+K_{E^{\prime}} \cdot \omega_{1} \cdot \Delta \delta=0
$$

$H \quad$ Inertia constant [MWs/MVA]

$\Delta \delta \quad$ Change in power angle $[\mathrm{rad}]$

$D \quad$ Damping constant [pu torque/pu speed]

$K_{E}$, Transient synchronizing torque coefficient [pu torque/rad]

From the swing equation, an expression (eqn. (6)) for the eigenvalues $\lambda_{1,2}$ describing the electromechanical swing mode for a single machine connected to stiff network can be derived (in this case the synchronous motor connected to the $50 \mathrm{~Hz}$ network):

$$
\lambda_{1,2}=-\frac{D}{4 H} \pm \sqrt{\left(\frac{D}{4 H}\right)^{2}-\frac{K_{E^{\prime}} \cdot \omega_{1}}{2 H}} .
$$

Eqns. (5) and (6) are valid for the converter in islanded operation, and need some modifications to be valid in interconnected operation when taking the damping and synchronizing torque of the generator into account as well.

\subsection{Linear analysis}

In this paper, a rotary converter in islanded operation is studied. The converter is connected to a weak $66 \mathrm{kV}$ three-phase utility grid having a short-circuit ratio of 250 MVA. There is no connection to other converters in the traction power system. The converter is feeding a $3.67 \mathrm{MW}$ resistive load over $60 \mathrm{~km}$ overhead contact line with the impedance of $(0.19+\mathrm{j} 0.21) \Omega / \mathrm{km}$. Including the catenary transmission losses this results in a loading close to the converter's rated load. Both machines are represented by $5^{\text {th }}$ order synchronous machine models.

The low frequency modes found by linearization of the converter at the operation point is shown in Table 1. Classification of the different mode is done 
by use of participation factors that indicate which state variables that participates mostly in the different modes. The most participating state variables in the electromechanical mode are the converter speed and motor power angle. Damping ratio for the electromechanical resonance is low but acceptable.

Table 1: $\quad$ Low frequency modes from linear analysis of rotary converter.

\begin{tabular}{|l|c|l|c|}
\hline No. & \multicolumn{1}{|c|}{ Eigenvalues } & Mode & Damping ratio \\
\hline 9,10 & $-4.67[1 / \mathrm{s}] \pm \mathrm{j} 3.66[\mathrm{~Hz}]$ & Generator exciter & $20 \%$ \\
\hline 11,12 & $-5.56[1 / \mathrm{s}] \pm \mathrm{j} 3.78[\mathrm{~Hz}]$ & Motor exciter & $23 \%$ \\
\hline 16,17 & $-0.49[1 / \mathrm{s}] \pm \mathrm{j} 1.39[\mathrm{~Hz}]$ & Electromechanical & $6 \%$ \\
\hline
\end{tabular}

\section{Advanced electrical rail vehicle}

\subsection{Introduction}

Almost all new electrical rail vehicles today utilize the advantages of the induction motor as traction motor (Östlund [12]). The motor speed and torque are controlled by use of a three-phase power electronic pulse-width modulated inverter. The motor inverter takes the required power from the vehicle internal DC link capacitor as shown in Figure 2. It is the task for the line inverter to modulate the DC link voltage into an AC voltage at main transformer low voltage side in amplitude and phase such that the resulting voltage drop over the transformer leads to the needed current to keep the DC link voltage at reference. All these components are controlled by an advanced computer based control system.

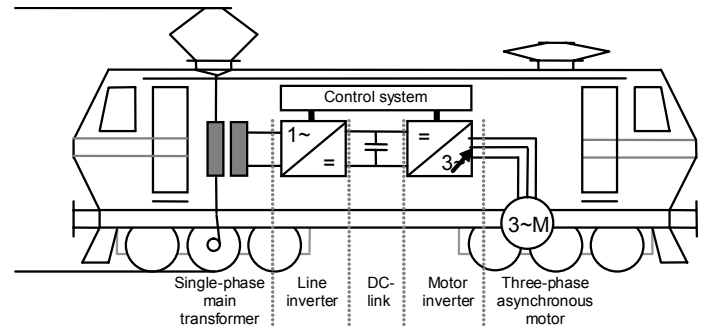

Figure 2: $\quad$ Principal sketch of an electrical advanced rail vehicle.

As both line and DC link capacitor exchange energy and because the power control can not be faster than the fundamental frequency, this result in a dynamical continuous feedback system (Menth and Meyer [1]).

\subsection{The model}

A model of such an advanced rail vehicle is made based on literature (Östlund [12] and Steimel [13]) and is given a thorough description in Danielsen et al. [7] for use in RMS simulations. Such RMS models of rail vehicles are not standard as most simulation studies in the rail vehicle industry are performed in 
instantaneous values. In RMS mode the model then comprises two major simplifications; the motor side is replaced by a resistor and the control system is analogous and continuous. Also, the reactive power consumption at current collector is controlled to be zero.

The line inverter control consists of the following controllers: Synchronizing controller (phase locked loop, $P L L$ ) to track the phase of the line voltage, DC link voltage controller $(V C)$ for active power control and AC current controller $(C C)$. The controllers are referred to a vehicle internal rotating reference frame, also known as vector control.

\subsection{Linear analysis}

The rail vehicle is operated at approximately half its rated power (3.67 MW) fed from an ideal voltage source through $60 \mathrm{~km}$ of overhead contact line. This means that in this sub-case, there is no rotary converter in the system.

The linear analysis identifies two low frequency modes. Their figures are shown in Table 2. The vehicle seems to be very well damped. Eigenvalues no. 12 and 13 will be subject to further studies as it describes the power oscillations.

In order to check the sensitivity of the interesting low frequency mode (eigenvalue 12 and 13), Figure 3 shows how the eigenvalue (positive imaginary part only as the pair is a complex conjugate) moves when different parameters for the locomotive are changed. The range of the change is from 0.5 to 2.0 times the original value, which is given in brackets in the legend. Arrows show the movement direction of the eigenvalue when the most influencing parameters are increased. The different parameters are explained in appendix.

Table 2: $\quad$ Low frequency modes from linear analysis of the vehicle.

\begin{tabular}{|l|c|l|c|}
\hline No. & Eigenvalues & Mode & Damping ratio \\
\hline 6,7 & $-12.9[1 / \mathrm{s}] \pm \mathrm{j} 7.04[\mathrm{~Hz}]$ & $\begin{array}{l}\text { AC current measurement, } \\
\text { AC voltage measurement }\end{array}$ & $28 \%$ \\
\hline 12,13 & $-5.66[1 / \mathrm{s}] \pm \mathrm{j} 3.31[\mathrm{~Hz}]$ & $\begin{array}{l}\text { VC, AC current meas., } \\
\text { DC link voltage, PLL }\end{array}$ & $26 \%$ \\
\hline
\end{tabular}

It can be noticed that reducing either the voltage controller integration time $(T I V C)$ or the current controller gain $(K P C C)$ will within the range alone move the eigenvalue into the right half plane and make the vehicle unstable. The voltage controller gain $(K P V C)$ has also influence on the mode. Changed DC link capacitance $(C)$ will move the eigenvalue in the same way as the inertia constant $H$ for synchronous machines in eqn. (6) as both express energy storages.

\section{System interaction}

\subsection{Unstable system}

The rotary converter and the rail vehicle are now combined into one system as shown in Figure 1. For the vehicle this leads to two changes even though the 
operation point is kept (as in section 4.3 and 5.3): the voltage source (both amplitude and phase) is no longer constant but will change as the converter oscillates, and the total inductance $L$ that the vehicle current controller has to change the line current in has increased. The latter change alone moves the eigenvalue to $-0.46[1 / \mathrm{s}] \pm \mathrm{j} 2.89[\mathrm{~Hz}]$, corresponding to a damping ratio of $2.5 \%$, if the impact of the generator automatic voltage regulator is not included.

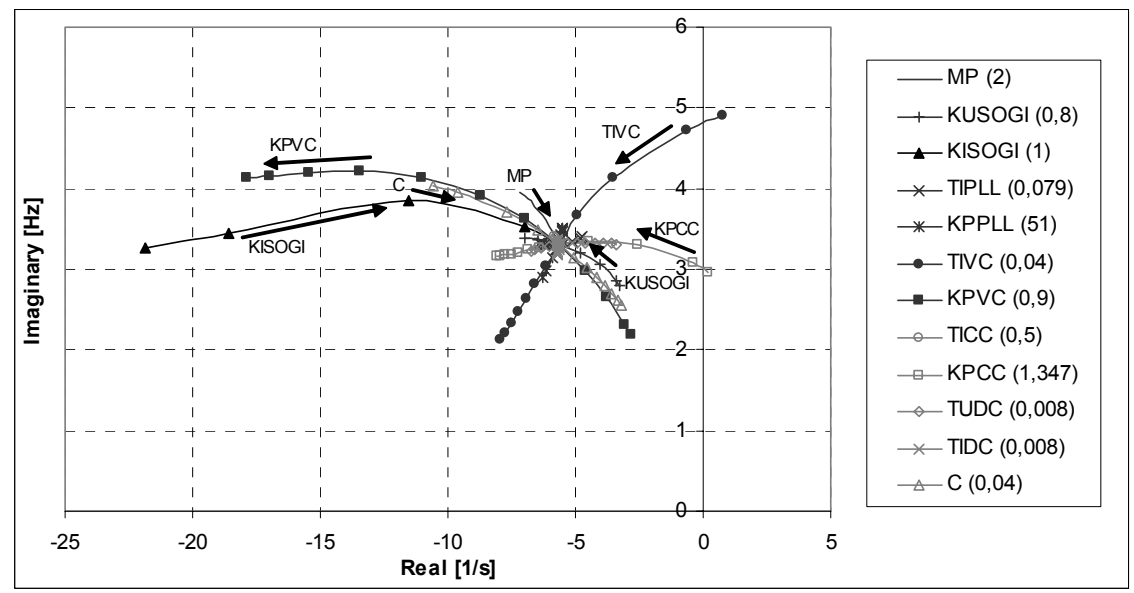

Figure 3: Root loci for the low frequency mode when parameters are changed.

When the rotary converter and the rail vehicle are combined, the system gets unstable. The low frequency modes are shown in Table 3. The vehicle DC link mode dominates and represents a root located in the right half plane. The electromechanical resonance mode is now practically without damping.

Table 3: Low frequency modes from linear analysis of traction power system.

\begin{tabular}{|l|c|l|c|}
\hline No. & Eigenvalues & Mode & Damping ratio \\
\hline 16,17 & $+4.28[1 / \mathrm{s}] \pm \mathrm{j} 3.34[\mathrm{~Hz}]$ & Vehicle DC link & $-20 \%$ \\
\hline 18,19 & $-4.62[1 / \mathrm{s}] \pm \mathrm{j} 3.81[\mathrm{~Hz}]$ & Generator exciter & $19 \%$ \\
\hline 20,21 & $-5.57[1 / \mathrm{s}] \pm \mathrm{j} 3.79[\mathrm{~Hz}]$ & Motor exciter & $23 \%$ \\
\hline 34,35 & $-0.03[1 / \mathrm{s}] \pm \mathrm{j} 1.41[\mathrm{~Hz}]$ & Rotary conv. electromech. & $0 \%$ \\
\hline
\end{tabular}

\subsection{Stability improvement}

By use of the linear analysis tool, again the influence of the vehicle control parameters on the different modes can be studied. Figure 4 shows the root loci when the parameters for the voltage and current controllers are changed. The arrows show the direction of move when the parameter value is increased.

It can be observed that $T I V C$ has a large influence on the vehicle mode, and an increase should move the root swiftly to the left, i.e. increasing it from $0.04 \mathrm{~s}$ 
to $0.10 \mathrm{~s}$ result in the eigenvalue $-1.36[1 / \mathrm{s}] \pm \mathrm{j} 2.75[\mathrm{~Hz}]$ which gives a damping ratio of $8 \%$. However, increase of $T I V C$ also has the impact of shifting the converter electromechanical resonance into the right half plane $(+0.07[1 / \mathrm{s}] \pm$ $\mathrm{j} 1.43[\mathrm{~Hz}])$ and the system is still unstable. Corresponding behaviour also applies to $K P C C$.

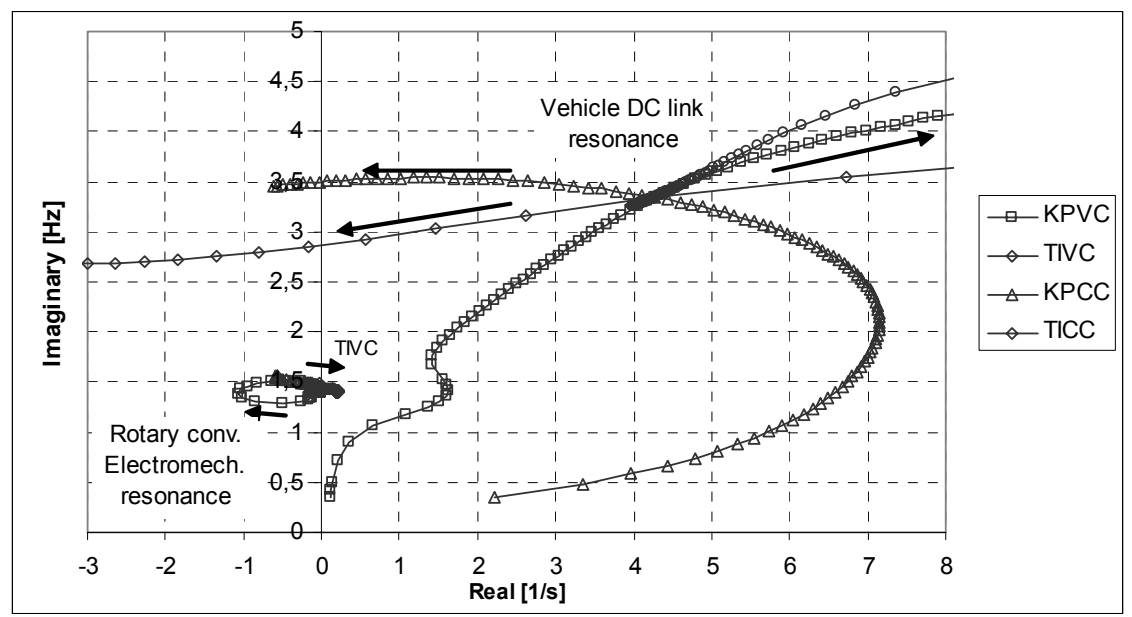

Figure 4: $\quad$ Root loci for the rotary converter and vehicle modes when vehicle control parameters are changed (0.0-2.0 for KPxx and 0.0-1.0 s for TIxx).

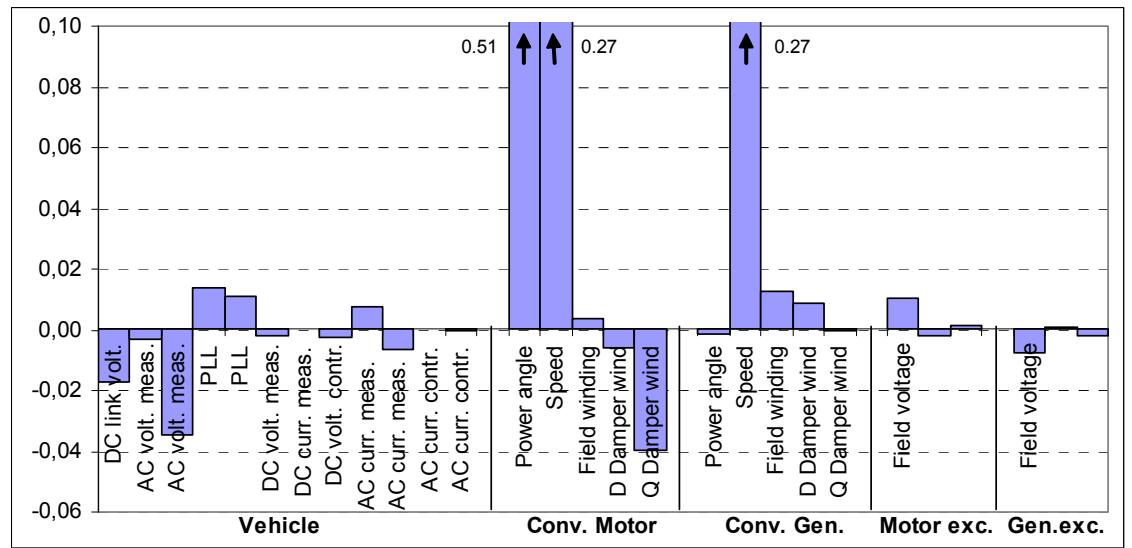

Figure 5: Real value of participation factors to show how state variables contribute to or damp the oscillation. 
It is hard to improve the damping of the electromechanical resonance mode, as it is not that sensitive to the present vehicle control parameters. It can though be observed that the vehicle is able to make the rotary converter unstable even though their resonance frequencies are different.

\subsection{Interaction with an unstable rotary converter}

To show how the rotary converter and the vehicle $(T I V C=0.10 \mathrm{~s})$ interact in this unstable system, the real part of the participation factors for the unstable electromechanical mode $+0.07[1 / \mathrm{s}] \pm \mathrm{j} 1.43[\mathrm{~Hz}]$ are shown in Figure 5. A positive value indicates that the state variable contributes to the oscillation, and a negative value indicates that the state variable oscillates in anti-phase and damps the oscillation. None of the vehicle controllers is found to have large contribution in the converter oscillation. The detailed results are difficult to interpret and should be object for more investigation.

\section{Concluding remarks}

In this paper linear analysis tools for studying low frequency power system oscillations have been applied to a traction power system to gain knowledge about the interaction between power supply infrastructure and rolling stock. The linear analysis clearly states the instability and provides information about which parts of the advanced components participate in the oscillation and which parameters to change for improvement. Here it is also observed that the electromechanical resonance of the rotary converter can be influenced by the vehicle even if the vehicle resonance frequency is located at another frequency. However, it is obvious that good results require adequate models that may be difficult to obtain, at least for the very complex advanced electrical rail vehicles.

\section{Appendix}

$M P$ is voltage dependency of motor load where value 2 is constant impedance characteristic. KUSOGI and KISOGI describe the filtering of line voltage and current, respectively. TIPLL and KPPLL are the integration time and gain of the $P L L$, respectively $T I V C$ and $K P V C$ are the integration time and gain for the DC link voltage controller, respectively $T I C C$ and $K P C C$ are the integration time and gain for the current controller, respectively $T U D C$ and $T I D C$ are the filter time constants for the DC voltage and current measurements, respectively $C$ is the DC link capacitor in Farad.

\section{Acknowledgements}

The authors want to thank Dr. Stefan Menth at Emkamatik in Switzerland for valuable discussions. The work presented in this paper is a part of a PhD study funded by the Norwegian National Rail Administration (Jernbaneverket). 


\section{References}

[1] Menth, S. \& Meyer, M., Low frequency power oscillations in electric railway systems, Elektrische Bahnen. 104(4), pp. 216-221, 2006.

[2] Danielsen, S. \& Toftevaag, T., Experiences with respect to low frequency instability from operation of advanced electrical rail vehicles in a traction power system with rotary converters. MET2007 8th International Conference "Drives And Supply Systems For Modern Electric Traction". Warsaw, Poland, pp. 51-57, 2007.

[3] Kundur, P., Power System Stability and Control, McGraw-Hill California, 1994.

[4] Fankhauser, H.R., Aneros, K., Edris, A.A. \& Torseng, S., Advanced simulation techniques for the analysis of power system dynamics, Computer Applications in Power, IEEE. 3(4), pp. 31-36, 1990.

[5] Eitzmann, M.A., Paserba, J.J., Undrill, J.M., Amicarella, C., Jones, A.L., Khalafalla, E.B. \& Liverant, W., Model development and stability assessment of the Amtrak $25 \mathrm{~Hz}$ traction system from New York to Washington DC. Railroad Conference. pp. 21-28, 1997.

[6] Slootweg, H., Persson, J., van Voorden, A.M., Paap, G.C. \& Kling, W., A Study of the Eigenvalue Analysis Capabilities of Power System Dynamics Simulation Software. 14th Power Systems Computation Conference 2002. Sevilla, Spain, pp. 2002.

[7] Danielsen, S., Fosso, O.B., Molinas, M., Suul, J.A. \& Toftevaag, T., Simplified models of a single-phase power electronic inverter for power system stability analysis - development and evaluation, Submitted to Electric Power System Research. pp. 2008.

[8] Harnefors, L., Modeling of Three-Phase Dynamic Systems Using Complex Transfer Functions and Transfer Matrices, Industrial Electronics, IEEE Transactions on. 54(4), pp. 2239-2248, 2007.

[9] Banverket/Jernbaneverket, Requirements on rolling stock in Norway and Sweden regarding EMC with the electrical infrastructure and coordination with the power supply and other vehicles (BVS 543.19300/JD 590), 2007

[10] Toftevaag, T. \& Pálsson, M.T., Low frequency oscillations in the Norwegian electric traction power supply caused by interaction between the supply system and propulsion machinery - analysis and consequences. MET'05 7th International Conference "Modern Electric Traction in Integrated XXIst Century Europe”. Warsaw, Poland, pp. 137-142, 2005.

[11] Biesenack, H., Theorie und Betriebsverhalten von Synchron-SynchronUmformern, Doktor Ingenieur thesis, Fakultät für Technik und Naturwissenschaft, Hochschule für Verkehrswesen "Friedrich List", 1972.

[12] Östlund, S., Elektrisk Traktion, Institutionen för Elektrotekniska System, Elektriska Maskiner och Effektelektronik, Kungliga Tekniska Högskolan, Stockholm, Sweden, 2001.

[13] Steimel, A., Electric Traction - Motive Power and Energy Supply, Oldenbourg Industrieverlag, München, 2008. 